\title{
Isolated attosecond pulses by self-compression in short gas-filled fibers
}

\author{
P. N. Anderson ${ }^{1}$, P. Horak ${ }^{1}$, J. G. Frey ${ }^{2}$, W. S. Brocklesby ${ }^{1}$ \\ 1. Optoelectronics Research Centre, University of Southampton, Southampton, SO17 1BJ, United Kingdom \\ 2. Chemistry, University of Southampton, Southampton, SO17 1BJ, United Kingdom
}

The intense few-cycle driving fields used to produce isolated attosecond pulses via high harmonic generation (HHG) [1] are typically delivered from conventional hollow fiber compressors. However, it has been suggested that individual attosecond pulses may be realized in low-pressure regions at the output of self-compressed filaments [2]. We follow a similar approach in an alternative geometry and present modelling of an elegant source of isolated attosecond pulses driven by a 40 fs near infrared (NIR) laser field. Here, ionization-induced self-compression and HHG happen in-situ within a short $(40 \mathrm{~mm})$ gas-filled fiber, and single 350 as pulses at a central wavelength of $13.5 \mathrm{~nm}$ are predicted at its exit.

We apply an advanced three-dimensional (3D) model based on a multimode-generalized nonlinear Schrödinger equation (MM-GNLSE) to simulate the propagation of intense driving pulses through gas-filled fibers [3]. Next, the time dependent Schrödinger equation (TDSE) describing the interaction of the electron wavefunction of single gas atoms with this driving field is solved numerically on a fine spatial grid, yielding the dipole accelerations. Lastly, XUV fields are constructed from these source terms and propagated through the fiber in the presence of both dispersion and loss. In order to accomplish these demanding calculations a massively parallel code has been developed and implemented on our 12000-core high performance computing resource. The design process for the source involved exploring the parameter space within our MM-GNLSE model to determine conditions where self-compressed pulses could be maintained over several $\mathrm{mm}$ with FWHM durations $<5$ fs (figure 1(a)). The XUV absorption length was also a consideration here and hence gas pressures were minimized as far as possible. When driven by a linearly polarized $800 \mu \mathrm{J}$ pulse, with a $40 \mathrm{fs}$ FWHM duration and a central wavelength of $790 \mathrm{~nm}$, our models predict isolated 350 as XUV pulses (figure 1(b)) to emerge from a $40 \mathrm{~mm}$ section of hollow fiber filled with $120 \mathrm{mbar}$ of argon. Additional analysis has shown that modifications to the spectral phase can reduce the duration of these pulses into the 100 as regime. As the selfcompression mechanism exploited within our source is versatile, work is ongoing to enable us to drive an isolated attosecond source with the 200-300 fs pulses delivered by state of the art fiber lasers.

(a)

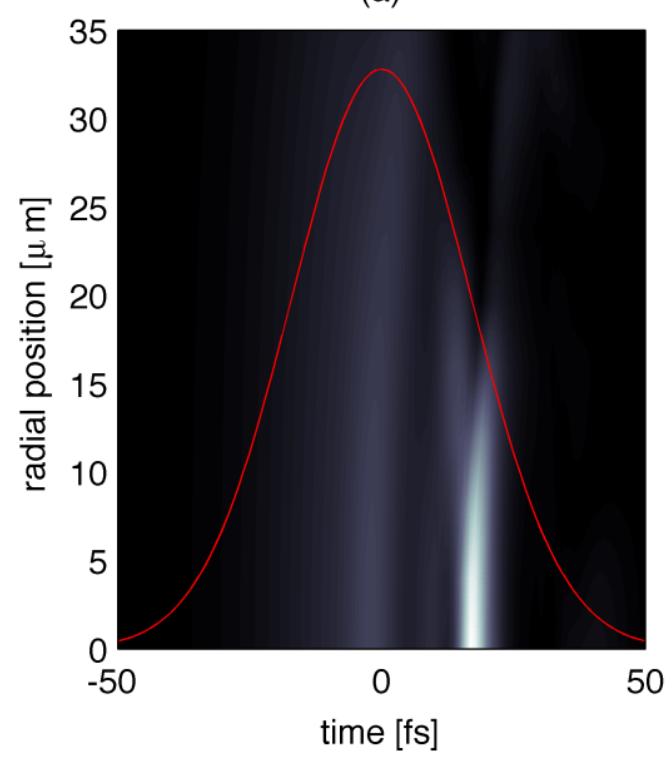

(b)

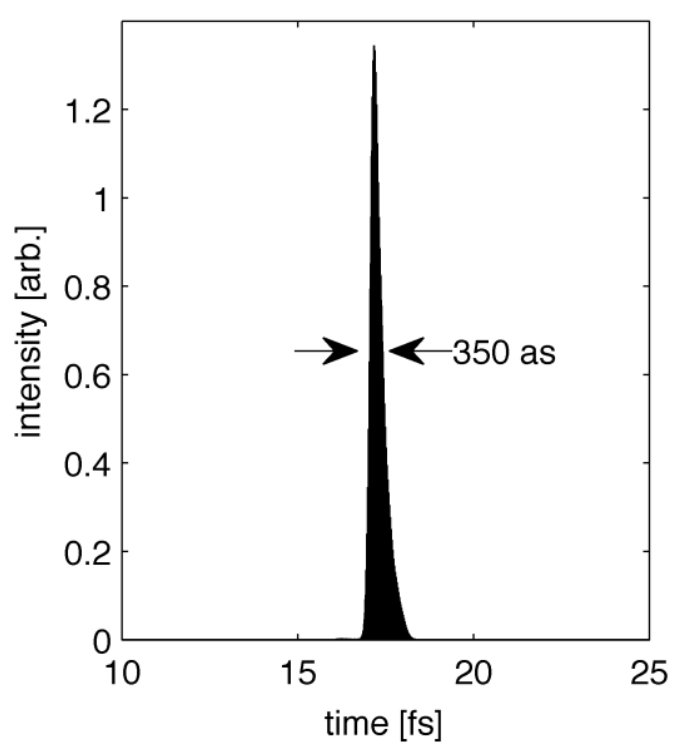

Fig. 1. (a) The spatio-temporal profile of the driving pulse towards the exit of a $40 \mathrm{~mm}$ length of hollow fiber filled with 120 mbar of argon. The solid curve illustrates the temporal profile ( $40 \mathrm{fs}$ FWHM) of the $800 \mu$ J NIR pulse launched. (b) The single attosecond pulse predicted to emerge from the fiber following appropriate spectral filtering. The isolation is attributed to the ionization-induced self-compression of the driving pulse to a FWHM duration of 4 fs.

\section{References}

[1] M. Hentschel, R. Kienberger, Ch. Spielmann, G. A. Reider, N. Milosevic, T. Brabec, P. Corkum, U. Heinzmann, M. Drescher, and F. Krausz, "Attosecond metrology," Nature 414, 509 (2001).

[2] H. S. Chakraborty, M. B. Gaarde and A. Couairon, "Single attosecond pulses from high harmonics driven by self-compressed filament," Opt. Lett. 31, 3662 (2006).

[3] R.T. Chapman, T. J. Butcher, P. Horak, F. Poletti, J. G. Frey and W. S. Brocklesby, "Modal effects on pump-pulse propagation in an Arfilled capillary" Opt. Express 18, 13279 (2010). 